Mots. Les langages du politique

$91 \mid 2009$

Que devient le pamphlet?

\title{
Les mutations du pamphlet dans la France contemporaine
}

Michel Hastings, Cédric Passard et Juliette Rennes

\section{OpenEdition}

\section{Journals}

Édition électronique

URL : https://journals.openedition.org/mots/19159

DOI : $10.4000 /$ mots. 19159

ISSN : 1960-6001

Éditeur

ENS Éditions

\section{Édition imprimée}

Date de publication : 30 novembre 2009

Pagination : 5-17

ISBN : 9782847881820

ISSN : 0243-6450

Référence électronique

Michel Hastings, Cédric Passard et Juliette Rennes, « Les mutations du pamphlet dans la France contemporaine ", Mots. Les langages du politique [En ligne], 91 | 2009, mis en ligne le 30 novembre 2011, consulté le 23 avril 2022. URL : http://journals.openedition.org/mots/19159 ; DOI : https:// doi.org/10.4000/mots. 19159 


\section{Les mutations du pamphlet dans la France contemporaine}

\section{Le pamphlet : le mot et la chose}

Souvent condamné à l'éphémère, le pamphlet se présente comme un écrit de circonstance peinant à survivre à la conjoncture particulière qui l'a vu ou qui l'a fait naitre. Aussi, l'histoire de la littérature pamphlétaire demeure encore mal connue et l'étymologie elle-même du mot pamphlet apparait incertaine (Angenot, 1982, p.372-377; Bellenger, 1984). Un rapide détour lexicographique permet de mettre en évidence l'émergence relativement tardive du pamphlet dans «l'ordre des livres » en France (Chartier, 1992) et le glissement de sens progressif du désignant vers sa signification actuelle. En effet, si pamflet fait son apparition dans la quatrième édition du Dictionnaire de l'Académie française en $1762^{1}$, sa définition - «mot anglais qui s'emploie quelquefois dans notre langue, ce qui signifie brochure» - prouve qu'il est alors encore peu usité et, surtout, n'indique aucune caractéristique le rattachant à la diatribe, ce qui tend à démontrer que pamphlet n'a pas encore acquis son sens contemporain, où prédomine l'idée de texte violent et agressif, d'esprit polémique et incisif. Ainsi, pamphlet a d'abord désigné la forme ou, plus précisément, le format d'un écrit plus que son contenu ${ }^{2}$. Au cours des $18^{\mathrm{e}}$ et $19^{\mathrm{e}}$ siècles, le terme prend une acception plus précise, associant, voire substituant de plus en plus à des critères morphologiques une visée - la dénonciation d'un personnage célèbre, d'une institution ou, plus généralement, d'un scandale - et un ton spécifique marqué par la véhémence ou la vitupération. Cette dimension intègre le pamphlet à la littérature de combat, comme en témoigne le célèbre Pamphlet des pamphlets paru en 1824, dans lequel

\footnotetext{
Université Lille Nord de France, Institut d'études politiques de Lille, CEPEN michel.hastings@iep.univ-lille2.fr Université Lille Nord de France, Institut d'études politiques de Lille, CEPEN cedric.passard@iep.univ-lille2.fr Université Lyon 2, Institut de la Communication.Juliette.Rennes@univ-lyon2.fr
}

1. D’après Yvonne Bellenger (1984), le premier emploi du terme en français est attesté en 1653 mais il s'y trouve présenté comme un mot anglais.

2. Le terme anglais pamphlet, dont est dérivé le mot français, conserve aujourd’hui le sens de brochure et ne contient pas l'idée d'écrit agressif. Il s'agit donc désormais d'un «faux ami ». 
Paul-Louis Courier le définit comme un «écrit tout plein de poison ». À cette fin, le pamphlet exploite différents procédés éristiques, notamment la satire, mais cette dernière ne lui est pas inhérente. Si le mot pamphlet ne s'impose donc, en France, qu'au $18^{\mathrm{e}}$ siècle et ne prend sa signification contemporaine qu'au 19', la chose se développe pourtant bien avant, comme l'avait analysé Yvonne Bellenger (1984, p. 89). Il n'est pas dans notre ambition de nous risquer, dans le cadre de cet article introductif, à une quête, peut-être d'ailleurs éperdue, des origines du genre. Il semble toutefois que l'éloquence pamphlétaire, qui mime la déclamation sur la voie publique, simule la harangue en plein air et convoque dans le discours écrit «l'imaginaire de l'oral» (Roussin, 2005, p.487), littéralise une tradition rhétorique de la colère qui a d'abord existé sous une forme oratoire, à la manière des Philippiques antiques. Les sources historiques et les modèles littéraires du pamphlet seraient ainsi à rechercher dans des formes anciennes de critique sociale (Walzer, 1996), et c'est avec le développement des grandes querelles politiques (guerre de Cent Ans et guerres civiles du $15^{\mathrm{e}}$ siècle) ou religieuses (Grand Schisme, guerres de religion du $16^{\mathrm{e}}$ siècle 3 ) que cette littérature connait son essor en France, même si certains auteurs peuvent aussi endosser la posture pamphlétaire de manière plus isolée, tel un Rutebeuf qui pourfend les ordres mendiants dès le $13^{\mathrm{e}}$ siècle. Cette époque inaugure une succession de grands moments pamphlétaires, associés à des périodes de conflits et d'agitation, ce qui a pour corollaire l'effacement relatif du pamphlet à d'autres périodes moins troublées. Cette succession de temps forts et d'éclipses périodiques de l'activité pamphlétaire suggère ainsi l'existence de configurations plus ou moins propices à son développement. Elle souligne, à l'évidence, la dimension critique que cette littérature présente à double titre : comme discours de combat et comme publication de crise. De fait, depuis la Fronde (Ducchini, 1978; Jouhaud, 1984) jusqu'à l'affaire Dreyfus au moins, les tumultes pamphlétaires coïncident avec les épisodes de tension sociale ou politique dont ils constituent un bon indicateur tout en contribuant souvent à les accentuer. Comme l'a bien montré Christian Jouhaud à propos des mazarinades, qu'il analyse comme des «scansions de l'action» (Jouhaud, 1984, p. 29), le caractère convulsif du pamphlet révèle sa sensibilité à l'évènement.

Cette histoire en pointillé du pamphlet se double d'une métamorphose de ses formes et de ses supports : libelle, brochure, tract, placard, chanson, journal, essai, voire roman, le discours pamphlétaire a connu bien des avatars. $\mathrm{Ni}$ jamais tout à fait le même ni tout à fait un autre, il dépend nécessairement non seulement de moyens techniques de diffusion et de reproduction disponibles en un temps et un lieu donnés, mais aussi d'un univers doxique et d'un

3. On notera à ce propos les similarités du discours de déploration du pamphlétaire avec la prédication et la parénèse luthériennes ou calvinistes qui s'énoncent sur un mode souvent agonistique (Collectif, 1983). 
horizon d'attente tout autant situés historiquement et culturellement. Cette plasticité, déconcertante pour le chercheur qui tenterait d'identifier une insaisissable substance du pamphlet, témoigne de la capacité de renouvellement des écrits pamphlétaires : leurs métamorphoses traduisent moins l'instabilité ou l'inconsistance du genre que ses propriétés d'adaptation à la variété des conditions sociohistoriques de leur production.

Aussi, contrairement aux apparences, les pamphlets ne sont des productions ni spontanées ni isolées; la mise en scène par les pamphlétaires de leur individualité, de leur solitude dans l'écriture et le combat ne doit pas occulter le caractère très souvent réticulaire de ces textes. Se développant généralement en grappes, les pamphlets participent, en ce sens, d'une modalité singulière d'action collective d'allure prédémocratique au regard des formes postérieures de prise de parole (manifestes, pétitions, grèves...). À cet égard, ils doivent être analysés comme des textes d'action, des textes "coup de force» ou «coup de poing ». Le pamphlétaire ne cherche pas, en effet, à convaincre ni même vraiment à persuader, mais à faire réagir et à créer l'évènement. La violence verbale du pamphlétaire semble s'enraciner, de ce point de vue, dans un horizon de performativité, dans une croyance en l'efficacité de la parole, en un pouvoir des mots.

Cependant, si le pamphlet se présente comme une arme de guerre qui engage une lutte symbolique sans limites contre son adversaire qu'il cherche à tuer, au moins métaphoriquement (Thomas, 1989; Duprat, 2002), toute la question est de savoir si cette guerre par les mots ne constitue qu'un fauxsemblant, ayant pour fonction de remplacer ou dépasser la violence physique ou si, au contraire, cette tonitruance du verbe se traduit effectivement dans l'action, dans un au-delà du langage. Autrement dit, peut-on prendre au pied de la lettre la logomachie d'un pamphlétaire qui se complait à théâtraliser sa rage? La violence du langage n'est parfois qu'un leurre. Les pamphlets, quand bien même ils se développent lors de périodes de remises en cause du pouvoir établi, sont loin d'être toujours révolutionnaires. Ils renvoient souvent, en réalité, à des jeux politiques complexes au sein du milieu dirigeant (Jouhaud, 1984 ; Popkin, 1989). Leur rôle dans les processus d'affaiblissement de l'autorité légitime et des systèmes de croyances dominants n'en reste pas moins déterminant. Au moment de la Révolution française, cette littérature marginale aurait ainsi contribué à saper le système idéologique de légitimation du régime (Darnton, 1985; Mornet, 1967). Sans être nécessairement révolutionnaires, donc, les pamphlets, en désacralisant les symboles monarchiques, en minant l'image des souverains, en tournant en dérision les rituels royaux, auraient concouru à la destruction de l'Ancien Régime. L'interprétation peut toutefois être renversée : n'est-ce pas parce que l'autorité symbolique et affective de la royauté était déjà entamée que les pamphlets ont pu connaitre une telle diffusion et un tel engouement? «Loin d'être les producteurs d'une telle 
rupture, ils en seraient donc les produits» (Chartier, 2000, p.126). Quoi qu'il en soit, les différentes flambées pamphlétaires accompagnent incontestablement les moments de crises politiques et les temps longs de la politisation. Avec la Révolution, survient cependant un changement décisif car la littérature pamphlétaire, qu'elle fût plus ou moins séditieuse, était jusque-là généralement réduite à la clandestinité. L'ouverture de l'espace public et la libération progressive de l'énonciation politique après la Révolution française lui permettent désormais d'abandonner progressivement ses habitudes contrebandières et de sortir de l'anonymat.

\section{Entre genre discursif et configuration historique. Retour sur l'analyse de Marc Angenot}

Marc Angenot, dans son ouvrage fondateur, La parole pamphlétaire. Typologie des discours modernes, paru en 1982, considère que ce modèle, en gestation depuis la période révolutionnaire, se déploie pleinement avec l'agonie du Second Empire, à partir de 1868, lorsqu'Henri Rochefort, à la faveur du nouveau régime de presse, publie sa célèbre Lanterne destinée, selon ses propres termes, à «éclairer les honnêtes gens et à pendre les malfaiteurs". Au cours des années qui suivent, l'installation de la République et la stabilisation de la démocratie donnent alors au pamphlet une liberté d'expression inédite, tandis que la diffusion de la presse et la massification du lectorat lui offrent une diffusion et un écho sans précédent. À l'intersection des différents champs - politique, littéraire, journalistique -, se dessine à cette époque un véritable espace pamphlétaire. Le pamphlet connaitrait, dans ce moment, son apogée ou son âge d'or, s'imposant comme forme spécifique et hégémonique d'intervention idéologique.

À travers cette étude, l'un des tours de force épistémologiques de Marc Angenot consiste à historiciser l'approche typologique qui caractérise classiquement les travaux d'argumentation. S'appuyant sur l'analyse des topoï fondée par Aristote et renouvelée par Chaïm Perelman et ses épigones, qui consiste à repérer et catégoriser des arguments, des raisonnements et des figures, propres à certains genres de discours et ajustés à certains types de publics (Aristote, 1990; Perelman, 1992), Marc Angenot met en relation les traits génériques qu'il identifie dans plusieurs centaines de pamphlets de 1868 à 1968, avec une configuration historique et idéologique marquée par l'émergence de l'intellectuel et la bipolarisation du champ politique et littéraire, la liberté de la presse et le développement des journaux populaires, la consolidation des grands récits idéologiques de la modernité et la structuration d'un imaginaire sociopolitique marqué par la hantise de la décadence. À partir de cette étude de cas, il montre ainsi très précisément comment un

8 Que devient le pamphlet? 
genre discursif relève en grande partie d'un ensemble de contraintes situationnelles provisoirement stabilisées dans des formes rhétoriques et des thématisations qui sont loin d'être transhistoriques. Ce genre historiquement déterminé est défini par Angenot comme "paradoxal». En effet, le pamphlet relève d'une posture qui consiste précisément à récuser les genres, les cadres et les idéologies en affichant son indépendance vis-à-vis des appareils partisans comme des institutions académiques, médiatiques et politiques. Le paradoxe est justement que cette posture est productrice de topoï et de figures polémiques récurrentes, qui font du pamphlet un genre à part entière, inscrit dans un marché éditorial et associé, pour les lecteurs, à un horizon d'attente esthétique, rhétorique et politique spécifique. Parmi les thématisations génériques décryptées par Marc Angenot, mentionnons :

- une certaine image de soi du pamphlétaire, autrement dit un ethos spécifique marqué par l'exotopie (se représentant comme une sorte de prophète en marge du système dominant et chargé d'en relever la turpitude, le pamphlétaire sursignifie le courage « viril» de sa solitude et de son verbe assassin);

- la mise en scène d'adversaires multiples et protéiformes reliés par des intérêts cachés et animés par une même logique, sinon un même dessein, selon la règle de l'ennemi unique toujours plus facile à désigner. La stratégie d'avilissement verbal de cet ennemi passe par des figures d'agression largement repérables, telles l'injure, l'usage de la scatologie et du calembour et la caricature du contre-discours;

- un récit sociopolitique structuré par un «noyau d'énoncés immuables»: le pamphlétaire part d'un scandale qui doit être absolument dénoncé, il n'a pour ce faire d'autre mandat que celui de la vérité bafouée; ce mandat, enfin, est à la fois urgent et vain puisqu'il est trop tard pour renverser le cours de la dégradation des valeurs (p.99 et suiv.). Le message du pamphlétaire se représente ainsi souvent comme une bouteille à la mer : la vérité qu'il clame, pourtant aveuglante, ne peut être entendue dans un monde dominé par l'imposture. Ce topos pamphlétaire du renversement des valeurs nourrit ainsi un sentiment d'impuissance qui rend ce genre discursif particulièrement propice aux idéologies du ressentiment;

- pessimiste, la vision du monde des pamphlets étudiés est appelée "crépusculaire» par Marc Angenot dès lors que le pamphlétaire postule une décadence généralisée et irréversible qui constitue le revers d'un Âge d'or propulsé dans un passé plus ou moins lointain et flou (" jadis», «lorsque j’étais enfant», "dans l'ancienne France») où viennent se loger toutes les valeurs investies de positivité (Liberté, Vérité, Moralité, Beauté, Probité, Ordre, Égalité, Démocratie, etc., selon les options idéologiques du pamphlétaire);

- enfin, cette fausse conscience dans le rapport à l'historicité conduit Marc Angenot à qualifier le pamphlet de genre tendanciellement «réactionnaire», non pas seulement parce que le pamphlétaire au sens strict réagitàl'imposture 
ambiante et «fait une scène», offrant le spectacle de sa propre colère, mais aussi parce qu'il est incapable d'investir des espoirs de transformation sociale dans les crises qui affectent la modernité.

Cette analyse rigoureuse, d'autant plus convaincante qu'elle porte sur un très vaste corpus, a incontestablement rempli son objectif de contribuer à une «typologie des discours modernes», comme l'indique son sous-titre. Si l'une des vertus de l'approche typologique est sa réappropriation possible à l'épreuve d'autres corpus, l'analyse de Marc Angenot a, de fait, alimenté nombre de travaux ultérieurs sur tel ou tel espace de la critique sociale (Boltanski, 1993; Haddad, 2004), mais également nourri l'histoire littéraire (Richter, 1984; Kapp, 1984, Declercq, Murat, Dangel, 2003; Ferret, 2007; Brissette, 2008) ainsi que l'histoire des idées, des idéologies ou des formations discursives (Taguieff, 1984; Ronzeaud, 1994; Rennes, 1999; Dumasy, Massol, 2001; Vrydaghs, 2007; Danblon, 2008). Dans cette dernière perspective, le pamphlet tel que décrit par Marc Angenot est considéré comme l'idéaltype d'une posture idéologique historiquement déterminée dont on retrouve certains ressorts cognitifs et politico-rhétoriques dans des discours ne relevant pas stricto sensu du genre pamphlétaire. Les contributions de ce numéro s'inspirent de cette étude pour la prolonger, la mettre en perspective, mais aussi la discuter sur certains points. Elles partent d'une question commune : qu'est devenu aujourd'hui ce genre pamphlétaire qui aurait connu en France son âge d'or sous la Troisième République? Comment sortir de l'aporie qui consiste à partir d'une définition du pamphlet comme un genre historiquement déterminé pour s'enfermer dans le constat de sa disparition? N'est-il pas également éclairant, pour comprendre les mutations contemporaines de l'espace de la critique sociale, de laisser de côté les définitions savantes du genre pamphlétaire pour partir des usages sociaux du terme pamphlet?

\section{L'archipel pamphlétaire aujourd'hui}

Plutôt que d'entonner le refrain du « Requiem pour un pamphlet», faisant écho aux paroles présomptueuses de Céline qui se voyait bien, à la fin de sa vie, en dernier chevalier d'une écriture pamphlétaire vouée à s'engloutir dans la «crasse modernité », il s'agit ici de s'interroger sur les recompositions contemporaines de l'espace pamphlétaire. Ce que les années Rochefort donnaient à voir, c'était une configuration singulière marquée par la figure héroïque d'un bretteur de plume, évoluant dans un contexte de nationalisation et de démocratisation de la politique, à travers le développement d'une parole dont les modalités n'avaient pas encore été entièrement pasteurisées par les normes juridiques. L'une des principales hypothèses de ce dossier de Mots. Les langages du politique est que le pamphlet continue bel et bien d'exister, mais 
qu'il ne parvient plus, comme à certaines périodes, à dessiner les frontières d'un espace critique légitime et institutionnalisé. Trois principales pistes d'explication peuvent être convoquées pour illustrer ce que nous appellerons le monde en archipel du pamphlet contemporain. Image d'une époque qui ne méconnait pas le pamphlet, mais ne le conçoit plus comme un espace autonome, bien identifiable par ses acteurs, ses lieux et ses interactions.

La première piste renvoie aux transformations des modalités d'intervention politique des intellectuels. Le pamphlétaire, tel que nous avions pris l'habitude de nous le représenter pendant un peu plus d'un siècle, constituait en quelque sorte une variation de la figure traditionnelle de l'intellectuel prophétique, que sa posture critique engageait à titre personnel sur des causes particulières au nom de valeurs universelles. Ce modèle d'engagement (Weber, 1995; Hollander, 2006), se décline à travers des figures historiques variées depuis le philosophe des Lumières, voire le libertin, jusqu'à l'écrivain dreyfusard en passant par le mage romantique et le publiciste de gazette. À la fois chasseur de scandales et gibier de vengeances, faisant reposer la légitimité de ses coups de gueule sur son autorité charismatique auprès d'un publiclectorat, le pamphlétaire fait partie de cette famille, même si son inscription se fait sur un mode mineur pour ne pas dire dévoyé : bien souvent gardien éructant d'un ordre moral et politique dont il souffre de voir les repères traditionnels se déliter, ses combats n'empruntent pas toujours, loin de là, les signes de la subversion. Or, il semblerait que ce modèle pamphlétaire soit aujourd'hui supplanté par de nouveaux modèles d'engagement des intellectuels (Sapiro, 2009). Les romanciers, qui alimentèrent largement la production pamphlétaire, faisant du pamphlet une affaire de style et d'écriture, tendent à être en retrait du débat politique, du moins dans l'espace public français4. En matière de dévoilement de l'imposture des puissants et des grands de ce monde, les hommes de lettres sont partiellement remplacés par de nouvelles figures d'artistes engagés, notamment cinéastes et documentaristes (Roussel, 2009) qui, dans leur écriture filmique, partagent avec les écrits pamphlétaires de l'époque précédente une forme d'outrance et de radicalité dénonciatrices volontiers identifiées comme "pamphlétaires» par la critique cinématographique5. Les hommes de lettres sont également devancés par la figure de l'«intellectuel spécifique» (Foucault, 1994) dont la légitimité des interventions doit moins aux propriétés prophétiques d'une parole solitaire qu'à la revendication d'un capital cognitif construit sur

4. Comme le remarque Gisèle Sapiro, cette « figure de l’intellectuel critique universaliste incarné par l'écrivain », si elle n'est plus prédominante en France, continue à exister sur la scène internationale «dans des cultures dotées d'une forte tradition lettrée». Sapiro cite Günter Grass en Allemagne, Orhan Pamuk en Turquie ou David Grossman en Israël (Sapiro, 2009, p. 31).

5. Sur les usages contemporains du terme pamphlet dans la critique de films et d'ouvrages, voir l'article de Christian Le Bart dans ce dossier. 
la spécialisation et la division du travail d'expertise que le détenteur utilise comme relais de la parole des dominés. Enfin, sans être totalement nouvelle, la figure de «l'intellectuel collectif» et parfois anonyme tend également à se développer au détriment de l'intellectuel prophétique qui monnayait dans ses interventions publiques le prestige de son nom propre. On peut mentionner en France le collectif Raison d'agir et la maison d'édition du même nom, le think-tank altermondialiste Fondation Copernic ou le site web de critique des médias Acrimed 6 , qui articulent des savoirs professionnels, théoriques et militants dans une perspective de critique sociale (Sapiro, 2009, p.30). Dans le même ordre d'idées, le média-activisme qui vise à effacer la «frontière entre producteurs d'informations bénévoles (militants, témoins) et professionnels (journalistes, experts)» (Cardon, Granjon, 2003, p. 71) contribue également à reconfigurer les supports et les modalités de la parole critique, en proposant sur le web des espaces de production et d'analyse de l'information, en dehors du périmètre d'intervention des grands médias et des élites militantes. C'est ainsi l'économie du crédit accordé à la parole critique qui se trouve bouleversée, à travers un renouvellement des répertoires d'action symbolique au profit de formules moins liées aux paradigmes de la singularité et de la vocation, qu'aux références fondées sur les connaissances scientifiques et les mobilisations collectives.

Une deuxième piste d'explication des transformations contemporaines de l'espace pamphlétaire renvoie à l'évolution des sensibilités sociales, aux modalités de leur inscription dans les normes juridiques, et plus largement à la légitimité croissante du recours au droit dans les cas d'outrage, d'injure et de diffamation. Le mythe de l'âge d'or du pamphlet s'est en effet construit autour de pratiques langagières marquées par l'outrance, la violence et la dégradation systématique de la cible visée, à la manière des caricatures. Injure, calomnie, dérision, bon mot, la parole pamphlétaire implique de mettre en scène à la fois la virtuosité de l'auteur et la blessure infligée à la victime. On comprend aisément que certains auteurs y aient vu la marque d'un ethos aristocratique, la permanence d'un univers symbolique marqué par le bel esprit et le persiflage, qui constituent l'arsenal discursif de distinction d'une classe qui trouve l'occasion de prolonger sa culture cynégétique et guerrière dans le verbe qui tue (De Baecque, 1996). Mais en un peu plus d'un siècle, les dynamiques de la démocratisation ont non seulement dévalué et marginalisé cet aristocratisme des conduites langagières, mais aussi déplacé les frontières du politiquement et du juridiquement acceptables, comme le montrent dans ce dossier Loïc Nicolas et Emmanuel de Jonge. En relation avec la montée du paradigme de l'égale dignité de tous les individus et les luttes des minorités pour la reconnaissance de leurs droits, les processus de pénalisation de l'in-

6. Action-CRItique-MEDias [URL: www.acrimed.org/], consulté le 23 juillet 2009 (site créé en 1996). 
sulte et de la diffamation raciale, sexiste et homophobe, en marche depuis les années soixante-dix, et plus largement les procès contre les propos stigmatisant tel ou tel groupe social, soumettent certaines traditions pamphlétaires à des contraintes inédites. Alors que l'horizon moral et normatif se reconstruit autour de demandes sociales et de revendications politiques de considération, de reconnaissance, elles-mêmes formulées à travers des procédures de victimisation, et de luttes contre ce qui peut être ressenti comme du mépris (Honneth, 2000, 2006), c'est tout un pan de la production pamphlétaire qui se trouve privé de ressources idéologiques et rhétoriques issues en particulier du dernier tiers du $19^{e}$ siècle.

Cependant, comme ce fut le cas à d'autres périodes et sur d'autres thématiques encadrées par d'autres formes de censure, les contraintes juridiques, l'injonction ethico-politique au respect des groupes discriminés et la police du langage sont à double tranchant. S'ils délimitent un périmètre strict de l'acceptable et du dicible, ces dispositifs de contrôle conduisent aussi à déplacer la parole pamphlétaire hostile aux minorités vers des supports moins exposés, tels les blogs, forums de discussion et autres formes de sites Internet, qui se trouvent, dès lors, érigés en espaces d'expression alternatifs, voire de résistance aux médias traditionnels accusés de contribuer à l'imposture du monde7. Ces dispositifs de contrôle nourrissent ainsi l'imaginaire conspiratoire et dessinent même de nouvelles raisons d'agir à certaines catégories de pamphlétaires prompts à dénoncer le «politiquement correct» et à se présenter à peu de frais comme les porte-voix courageux de ce qui se dit tout bas mais ne peut s'énoncer publiquement ${ }^{8}$. Réhabilitant, à travers des processus de stéréotypage de leurs adversaires, les lieux communs sur telle ou telle catégorie sociale ou politique historiquement stigmatisée et érigée par les pamphlétaires en «lobby» tout puissant (les juifs, les sionistes, les islamogauchistes, les féministes, les lesbiennes, les pédés, etc.), ils s'opposent alors frontalement à la fois à la démarche sociologique de dénaturalisation des groupes sociaux et au discours normatif des droits humains et de la nondiscrimination.

Face à de telles postures, la réception des grands médias est ambigüe. Comme l'analyse Christian Le Bart dans ce dossier, les journalistes chargés des rubriques consacrées à la littérature d'idées tendent à employer le terme

7. Voir par exemple, parmi beaucoup d'autres, le site www.plumenclume.net, qui entend «taper sur les sionistes jusqu'à plus soif» pour «sauver la liberté d'expression, sur laquelle les sionistes sévissent, puisqu'ils ont fait voter des lois pour leur assurer l'impunité, telle en France, la loi Gayssot, de 1990 » (citation relevée le 16 juillet 2009).

8. Certains noms de sites sont éloquents: Onnouscachetout, À contre-courant, Conspiration.CC... Ils évoquent parfois d'ailleurs, de manière significative, la presse pamphlétaire de la fin du $19^{\mathrm{e}}$ siècle, comme les sites antisémites La voix des opprimés ou Les Intransigeants. Autre site antisémite, Lesogres.org prétend «parler de tout ce dont les marchands ne parlent pas, ou les contredire lorsqu'ils mentent». 
pamphlet pour signifier un double discrédit : celui d'une transgression morale tout d'abord, qui voit l'auteur commettre une sorte de faute de goût, à la fois ridicule et désuète; celui, ensuite, d'une analyse dont la portée se trouve affaiblie voire anéantie par les seuls effets d'une écriture jugée excessivement polémique. Mais en même temps, de nombreux formats médiatiques - tribunes libres des grands quotidiens, émissions radiophoniques et surtout télévisées consacrées aux débats de société, talk-shows, etc. - sont à la recherche de positions tranchées, provocantes, transgressives qui garantissent le spectacle de l'affrontement verbal, avec ses violences, ses surprises et ses coups bas. Cette demande médiatique pour la posture pamphlétaire garantit à certains un succès, sinon éditorial, du moins télévisuel lorsqu'il s'agit de révéler, de plateaux en plateaux et face à des opposants ad hoc chargés d'alimenter la polémique, tel ou tel complot politique, tel ou tel renversement des rôles sexués, telle ou telle invasion de "nouveaux barbares».

De façon plus récente et relevant d'une autre logique qui concerne davantage les propos pamphlétaires envers les autorités qu'envers les minorités, les procédures de contrôle étatique de l'outrage ont des effets tout aussi ambigus, entre étouffement et radicalisation de la parole critique. On pense notamment à la multiplication de poursuites judiciaires envers des groupes de rap ou envers des individus pour outrage aux personnes dépositaires de l'autorité publique, au drapeau tricolore ou à l'hymne national, ou encore à la proposition de loi présentée en septembre 2005 par des députés de la majorité présidentielle et visant à instaurer un «délit d'atteinte à la dignité de la France et de l'État», étudiées ici par Cédric Passard. Sans mobiliser les mêmes acteurs ni les mêmes enjeux politiques que la lutte contre les propos discriminatoires, cette dynamique de normalisation et de contention de la parole publique contribue elle aussi à recomposer le périmètre de l'espace pamphlétaire contemporain.

Les modes actuels de recomposition du pamphlet nous semblent enfin redevables de la perte de légitimité des institutions d'autorité et de la crise des régimes de vérité, selon les propositions de Michel Hastings dans ce dossier. Le pamphlétaire incarnait, dans la seconde moitié du $19^{\mathrm{e}}$ siècle, une sorte de "maître de la vérité» (Détienne, 1981). Personnage emblématique d'un rôle social critique du scandale, le pamphlétaire s'apparentait alors à l'archétype du "témoin ambassadeur», nouvelle manière de faire du journaliste celui qui expose les faits à la lumière et qui déjoue la part d'ombre et de mystère des évènements (Muhlmann, 2004). En ce sens, on peut comprendre l'importance acquise à cette époque par le genre pamphlétaire en référence à la prégnance encore très forte des « institutions de vérité » (Église, École, État), et plus généralement aux modes autoritaires d'énonciation des vérités comme la religion bien sûr, mais aussi le positivisme des sciences sociales émergentes (Lepenies, 1990). Des phénomènes relevant de différentes logiques, comme la montée du paradigme constructiviste en sciences 
sociales, le discrédit de certaines institutions d'autorité et la crise des «religions séculières » de la modernité (Hartog, 2003; Lasch, 2002), ont affecté les mécanismes de l'écriture pamphlétaire et rendu beaucoup plus ardue la prétention à parler au nom d'une vérité supérieure. Dit autrement, le pamphlétaire de l'âge d'or serait victime du soupçon qui se porte sur les différentes formes de vérité : qu'il s'agisse de l'histoire et des récits longtemps présentés comme exprimant la vérité du passé, désormais dénoncés comme tendancieux, idéologiques ou apologétiques, qu'il s'agisse également des valeurs morales au nom desquelles le pamphlétaire construit son indignation, et qui n'offrent plus la même assise consensuelle que lors des deux derniers siècles. Mais, une fois encore, le paradoxe est que cette crise des régimes de vérité qui traverse aussi bien les sciences exactes et humaines (Williams, 2006) que l'espace du débat public, alimente à son tour une partie des productions pamphlétaires contemporaines qui voient dans ces transformations de l'espace de la critique sociale un irrémédiable «dépérissement de la politique»9.

Ily a donc bien une part indémodable du pamphlet. Elle réside non pas dans une quelconque permanence qui favoriserait les définitions préalables, mais dans l'adaptabilité constante d'un genre politico-littéraire qui épouse l'espace public de la critique et y transgresse par l'écriture les normes de la juste mesure. Plasticité de ses formes historiques, pluralité de ses usages sociaux et culturels : le genre semble constamment déjouer les catégories toutes faites. Comme l'a analysé Jacques Bouveresse, le pamphlet préfère endosser cette propriété singulière que Karl Kraus attribuait déjà à la satire : exagérer le présent afin d'essayer désespérément d'empêcher que la réalité lui donne demain raison.

\section{Références}

ANG ENOT Marc, 1995 [1982], La parole pamphlétaire. Typologie des discours modernes, Paris, Payot, Rivages.

ARISTOTE, 1990, Organon V. Les topiques, Paris, Vrin.

BAECQUe Antoine (DE), 1996, "Les ridicules de l'homme nouveau. Un groupe de satiristes sous la Révolution», Mots. Les langages du politique, $\mathrm{n}^{\circ} 48$, Caricatures politiques, p.15-32.

BeLLENGER Yvonne, 1984, "Le pamphlet avant le pamphlet. Le mot et la chose», Paris, Cahiers de l'Association internationale des études françaises, vol. 36, $\mathrm{n}^{\circ} 1$.

BolTANSKI Luc, 1993, La souffrance à distance, Paris, Métailié.

Bouveresse Jacques, 2007, Satire et prophétie. Les voix de Karl Kraus, Paris, Agone.

BRISSETTE Pascal, 2005, La malédiction littéraire. Du poète crotté au génie malheureux, Montréal, Presses de l'université de Montréal.

9. Sur ce discours, voir les analyses de Myriam Revault d'Allonnes (2002). 
CARdon Dominique, Granjon Fabien, 2003, «Peut-on selibérerdesformats médiatiques? Le mouvement alter-mondialisation et l'Internet», Mouvements, $\mathrm{n}^{\circ} 25$.

CHARTIER Roger, 1991, Les origines culturelles de la Révolution française, Paris, Le Seuil.

- 1992, L'ordre des livres. Lecteurs, auteurs, bibliothèques en Europe entre xive et xVIII siècle, Aix-en-Provence, Alinea.

ColleCtIF, 1983, Le pamphlet en France au xvie siècle, Paris, Publications de l’École nationale supérieure de jeunes filles (Cahiers V.-L. Saulnier).

DANBLon Emmanuelle, 2008, «Figures de la transgression du lien social dans le pamphlet», Émotions et discours. L'usage des passions dans la langue, M. Rinn éd., Rennes, Presses universitaires de Rennes, p. 99-111.

DARNTON Robert, 1985, Édition et sédition : l'univers de la littérature clandestine au XVIII' siècle, Paris, Sorbonne.

DeCLercq Gilles, Murat Michel, Dangel Jacqueline, 2003, La parole polémique, Paris, Champion.

Detienne Marcel, 1981, Les maîtres de vérité dans la Grèce antique, Paris, Maspero.

DuccıNI Hélène, 1978, «Regard sur la littérature pamphlétaire en France au XVIIe siècle », Revue historique, $\mathrm{n}^{\circ} 528$.

DumAsy Lise et MASSOL Chantal, 2001, Pamphlet, utopie, manifeste (xIXe - xxe siècles), Paris, L'Harmattan.

DUPRAT Annie, 2002, Les rois de papier. La caricature de Henri III à Louis XVI, Paris, Belin.

FERRET Olivier, 2007, La fureur de nuire. Échanges pamphlétaires entre philosophes et antiphilosophes (1750-1770), Oxford, University of Oxford, Studies on Voltaire and the Eighteenth Century, Voltaire Foundation.

Foucault Michel, 1994 [1984], «L'intellectuel et ses pouvoirs », Dits et écrits, t. IV, Paris, Gallimard, p. 676-677.

HAdDAD Galit, 2004, "La querelle du "défaitisme" en 1917 ", Mots. Les langages du politique, $\mathrm{n}^{\circ} 76$, Guerres et paix. Débats, combats, polémiques.

HARTOG François, 2003, Régimes d'historicité, Paris, Le Seuil.

Hollander Paul, 2006, The End of Commitment. Intellectuals, Revolutionaries and Political Morality, Chicago, Dee.

Honneth Axel, 2000, La lutte pour la reconnaissance, Paris, Le Cerf.

— 2006, La société du mépris, Paris, La Découverte.

Jounaud Christian, 1985, Mazarinades. La Fronde des mots, Paris, Aubier.

KAPP Volker, 1984, "Satire et injure au XVIIIe siècle. Le conflit entre la morale et la politique dans le débat sur les libelles ", Cahiers de l'Association internationale des études françaises, vol. 36, $\mathrm{n}^{\circ}$ 1, p. 155-165.

LASH Christopher, 2002, Le seul et vrai paradis. Une histoire de l'idéologie du progrès et de ses critiques, Paris, Climats.

LEPENIES Wolf, 1990, Les trois cultures, Paris, MSH.

MORNET Daniel, 1967, Les origines intellectuelles de la Révolution française, Paris, Armand Colin.

Muhlmann Géraldine, 2004, Une histoire politique du journalisme, Paris, Presses universitaires de France. 
Perelman Chaïm, Olbrechts-Tyteca Lucie, 1992 [1958], Traité de l'argumentation. La nouvelle rhétorique, Bruxelles, Presses de l'université de Bruxelles.

POPKIN Jeremy, 1989, "Pamphlet journalism at the end of the old regime», Eighteenth Century Studies, vol. 22, $\mathrm{n}^{\circ} 3$.

RENNES Juliette, 1999, "L'idée de décadence dans les pamphlets d'extrême droite des années 1930 ", Mots. Les langages du politique, $\mathrm{n}^{\circ} 58$, Argumentations d'extrême droite.

Revault D'Allonnes Myriam, 2002, Le dépérissement de la politique. Généalogie d'un lieu commun, Paris, Flammarion.

RICHTER Bodo L. O., 1984, «François de Belleforest. Un des pamphlétaires fulminants du xvı siècle», Cahiers de l'Association internationale des études françaises, vol. $36, n^{\circ} 1$, p. $97-110$.

RONZEAUd Pierre, 1994, «Un genre non classique. Le pamphlet », Littératures classiques, $\mathrm{n}^{\circ} 19$, Le classicisme.

RouSSEL Violaine, 2009, "Parler au nom des publics. Sociologie des prétentions à représenter de célébrités américaines dans le contexte de la guerre en Irak», Sociétés contemporaines, $\mathrm{n}^{\circ} 74$.

Roussın Philippe, 2005, Misère de la littérature, terreur de l'histoire, Paris, Gallimard.

SAPIRO Gisèle, 2009, «Modèles d'intervention politique des intellectuels. Le cas français », Actes de la recherche en sciences sociales, $\mathrm{n}^{\circ}$ 176-177, p. 8-31.

TAGUieff Pierre-André, 1984, «La rhétorique du national-populisme», Mots. Les langages du politique, octobre, $\mathrm{n}^{\circ} 9$.

ThomAs Chantal, 2003, La Reine scélérate. Marie-Antoinette dans les pamphlets, Paris, Le Seuil.

VRYDAGHS David, 2007, «La variation idéologique des systèmes d'adhésion », COnTEXTES, $\mathrm{n}^{\circ} 2$ [En ligne], [URL: http://contextes.revues.org/index204.html], consultéle 23 juillet 2009.

Walzer Michael, 1996, La critique sociale au xxe siècle. Solitude et solidarité, Paris, Métailié.

Weber Max, 1995, Économie et société, Paris, Plon.

Williams Bernard, 2006, Vérité et véracité. Essai de généalogie, Paris, Gallimard. 